\title{
Testing and experimental characterization of a linear permanent magnet actuator for active vehicle suspension
}

\author{
Jiabin Wang* and Weiya Wang *
}

\begin{abstract}
This paper describes the testing and experimental characterization of a linear permanent magnet actuator, which is designed and developed for active vehicle suspension, under both static and dynamic conditions. Since the active suspension unit operates over a wide forcevelocity range with varying duty ratios, it is essential to establish an effective thermal model which can be used for assessing temperature rise of the actuator under various operating conditions. The temperature rise of the actuator is measured and the results are compared with the prediction by the derived transient thermal model. It is shown that the measured actuator parameters and characteristics are closed to their predicted values. The linear actuator is controlled by a dSPACE system via a three phase inverter and its velocity tracking performance is presented.
\end{abstract}

Keywords: Linear motors, Permanent magnet motors, Active vehicle suspension

\section{Introduction}

Traditional designs of automotive suspension with passive components have been a compromise between the three conflicting demands of road holding, load carrying, and passenger comfort. In contrast to passive systems, active suspension systems can adjust their dynamic characteristics in response to varying road conditions, and offer superior handling, road feel and responsiveness as well as roll stability and safety without compromising ride quality. Although electro-hydraulic active suspension has been extensively researched and developed [1]-[3], it does not possess the bandwidth of a fully electric system, and thus compromise the anticipated performance improvements. In addition, the high energy consumption and assembly costs have proven disadvantageous [4].

Linear electromagnetic actuator (LEA) based active suspension has superior controllability and bandwidth, provides shock load isolation between vehicle chassis and wheel due to absence of any mechanical transmission, and therefore has a much great potential [5], [6].

It also has the ability to recover energy that is dissipated in the shock absorber in the passive systems [7]-[8], and results in a much more energy efficient suspension system. A

* Department of Electronic and Electrical Engineering, the University

of Sheffield, Mappin Street, Sheffield, S1 3JD UK

(j.b.wang@sheffield.ac.uk; weiya.wang@sheffield.ac.uk)

Received 13 October 2012; Accepted 10 November2012 9-slot, 10-pole quasi-Halbach magnetized tubular machine with modular winding, Fig. 1, has been developed for active suspension [9]-[11].

Each pole of the magnetic armature comprises of one radially and one axially magnetized ring magnet. The salient feature of the quasi-Halbach magnetization is that the axially magnetized magnets essentially provide a return path for the radial air-gap flux, and hence, the flux in the inner bore is relatively small.

As a result, the use of a very thin ferromagnetic tube or even a non-magnetic tube on which to mount the magnets will not significantly compromise the thrust force capability. This is conducive to reducing unsprung mass and hence enhancing the dynamic capability of active suspension.

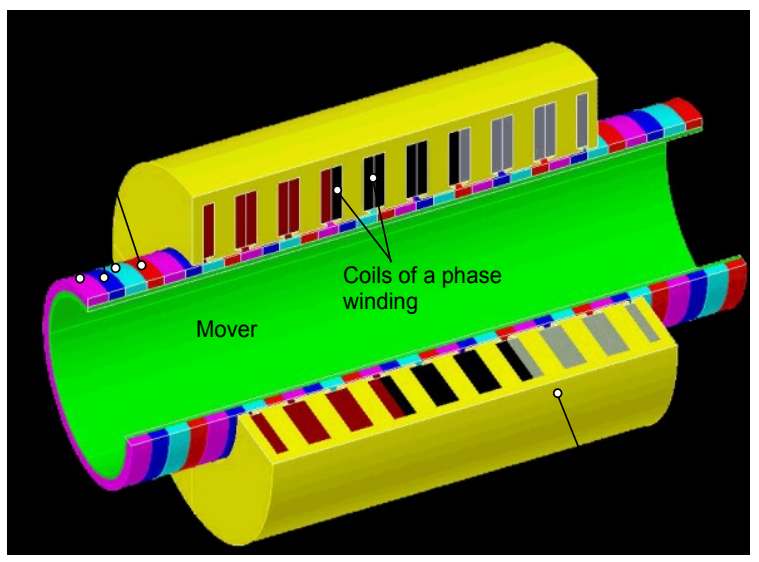

Fig.1. Schematic of 9-slot, 10-active pole tubular PM machine with quasi-Halbach magnetization 


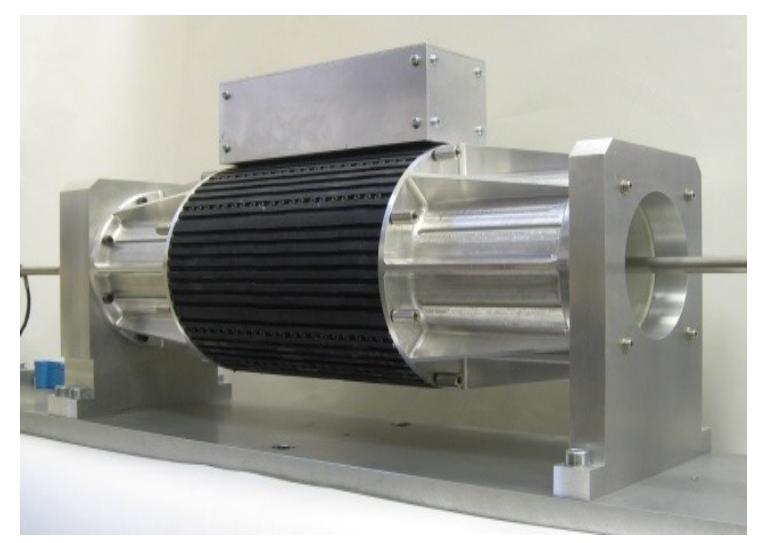

Fig. 2. Prototype LEA on test rig

A prototype tubular PM machine optimized for maximum force capability, as shown in Fig. 2, has been constructed and integrated into a compact active suspension strut unit, Fig. 3. This paper describes the testing and experimental characterization of the actuator and suspension unit under both static and dynamic conditions.

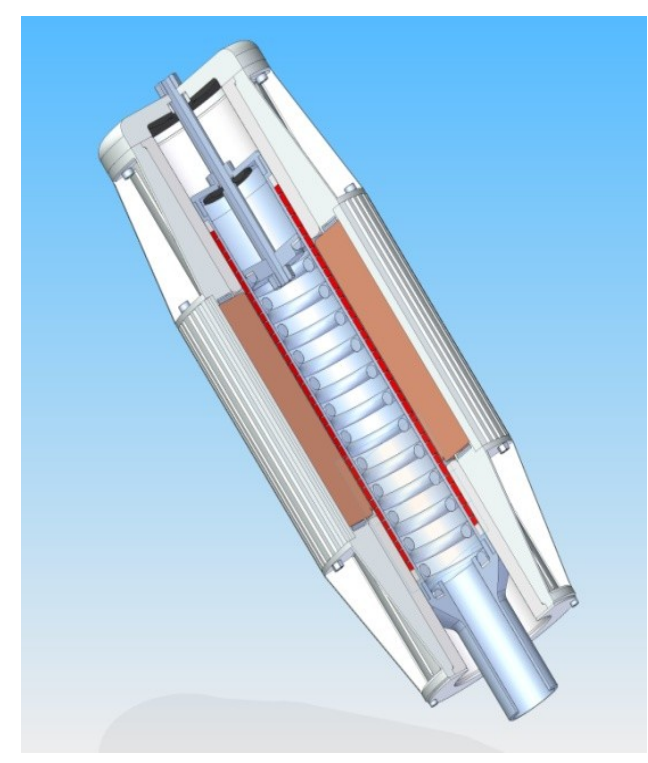

Fig. 3. Schematic of integrated design of LEA based active vehicle suspension unit

\section{Static Measurements}

The design parameters and specifications of the linear actuator are given in Table 1 . The actuator operates with $42 \mathrm{~V}$ DC link voltage and outputs a peak force of $5000 \mathrm{~N}$ at phase current of 208A peak. The continuous force capability is $2200 \mathrm{~N}$ with a forced air cooling at a minimum flow rate of $8 \mathrm{~m} / \mathrm{s}$, which corresponds to a vehicle speed of $\sim 17.5$ miles per hour.

Table 1. Specification, design parameters and thermal conditions

\begin{tabular}{|l|c|}
\hline \hline Outer stator diameter & $0.180(\mathrm{~m})$ \\
\hline Active length & $0.200(\mathrm{~m})$ \\
\hline Air-gap length & $0.001(\mathrm{~m})$ \\
\hline Stroke & $\pm 0.065(\mathrm{~m})$ \\
\hline Mover radius & $0.099(\mathrm{~m})$ \\
\hline Number of poles & 10 \\
\hline Pole-pitch, $\tau_{p}$ & $20(\mathrm{~mm})$ \\
\hline Number of slots & 9 \\
\hline Slot-pitch & $22.2(\mathrm{~mm})$ \\
\hline Magnet thickness & $5.0(\mathrm{~mm})$ \\
\hline Maximum temperature rise & $120^{\circ} \mathrm{C}$ \\
\hline Ambient temperature & $60{ }^{0} \mathrm{C}$ \\
\hline Thermal convection coefficient & $40 \mathrm{~W} /{ }^{0} \mathrm{Cm}{ }^{2}$ \\
\hline Remanence of magnets & $1.15(\mathrm{~T})$ \\
\hline DC link voltage & $42(\mathrm{~V})$ \\
\hline Continuous force & $2250(\mathrm{~N})$ \\
\hline Peak force & $5000(\mathrm{~N})$ \\
\hline Continuous phase current & $80.5(\mathrm{~A} \mathrm{peak})$ \\
\hline Maximum phase current & $208(\mathrm{~A} \mathrm{peak})$ \\
\hline \hline
\end{tabular}

The phase resistance and phase-self inductance were measured at the room temperature of $23{ }^{\circ} \mathrm{C}$ and the results are compared with the prediction in Table 2 . As can be seen, the measured phase resistance is about $4.3 \%$ larger than the predicted value, mainly due to the resistance of the connection leads between the phase coils, which are not included in the prediction. Slight difference in the estimate of the average length of the coils may also contribute the error in prediction. By comparing the measured and predicted phase self-inductances, one can see that a large difference of $\sim 16.6 \%$ exists. This is attributable to the fact that measurement was taken place with $50 \mathrm{~Hz}$ excitations. Since the stator core is constructed using segmented pieces of solid iron [10], there will be induced eddy current in the stator as a result of $50 \mathrm{~Hz}$ excitation. The induced eddy current reduces the phase flux linkage and hence the self-inductance.

Table 2. Comparison of measured and predicted phase resistance and inductance

\begin{tabular}{|c|c|c|c|}
\hline & Measured & Predicated & Error $(\%)$ \\
\hline \multicolumn{4}{|c|}{ Resistance (at $20^{\circ} \mathrm{C}, \mathrm{m} \Omega$ ) } \\
\hline Phase A & 35.9 & 34.8 & +3.2 \\
\hline Phase B & 37.2 & 34.8 & +6.9 \\
\hline Phase C & 35.8 & 34.8 & +2.9 \\
\hline Average & 36.3 & 34.8 & +4.3 \\
\hline \multicolumn{4}{|c|}{ Inductance $(\mathrm{mH}$, measured at $50 \mathrm{~Hz})$} \\
\hline Phase A & 0.605 & 0.719 & -15.9 \\
\hline Phase B & 0.585 & 0.719 & -18.3 \\
\hline Phase $\mathrm{C}$ & 0.610 & 0.719 & -15.2 \\
\hline Average & 0.600 & 0.799 & -16.6 \\
\hline
\end{tabular}

The variations of phase and line-to-line flux linkages with the mover axial displacement were measured by a flux meter. The mover displacement was recorded by a linear variable 
differential transformer (LVDT). The results are shown in Fig. 4. It is observed that the waveforms of phase or line-toline flux linkages are virtually identical with a phase shift of $\sim 120^{\circ}$ electrical degree between each other. There is, however, a small difference of less $1.5 \%$ in the magnitude of flux linkage between phase $\mathrm{A}$ and phase $\mathrm{B}$ and between phase $\mathrm{B}$ and phase $\mathrm{C}$.

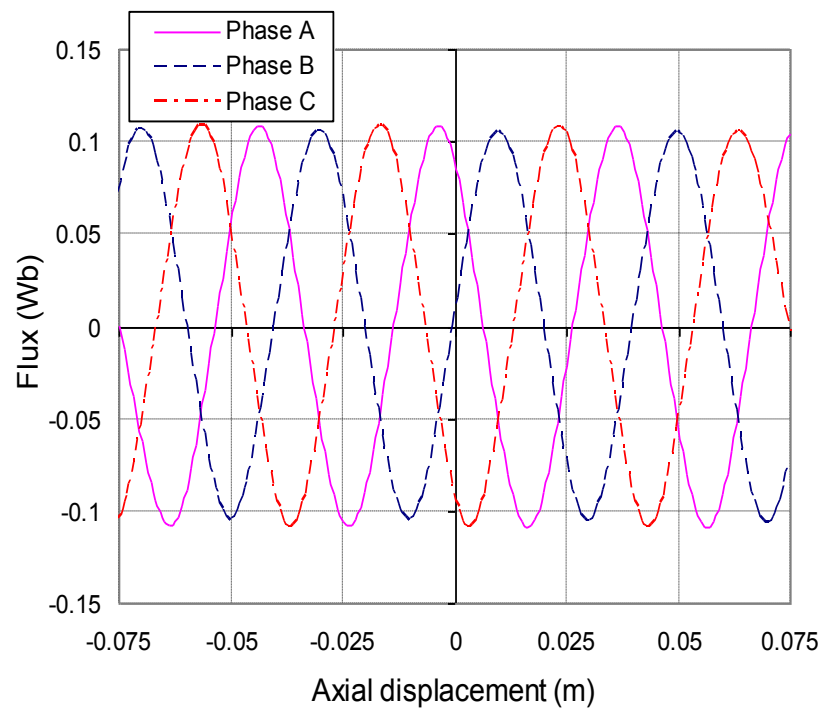

(a) phase flux linkages

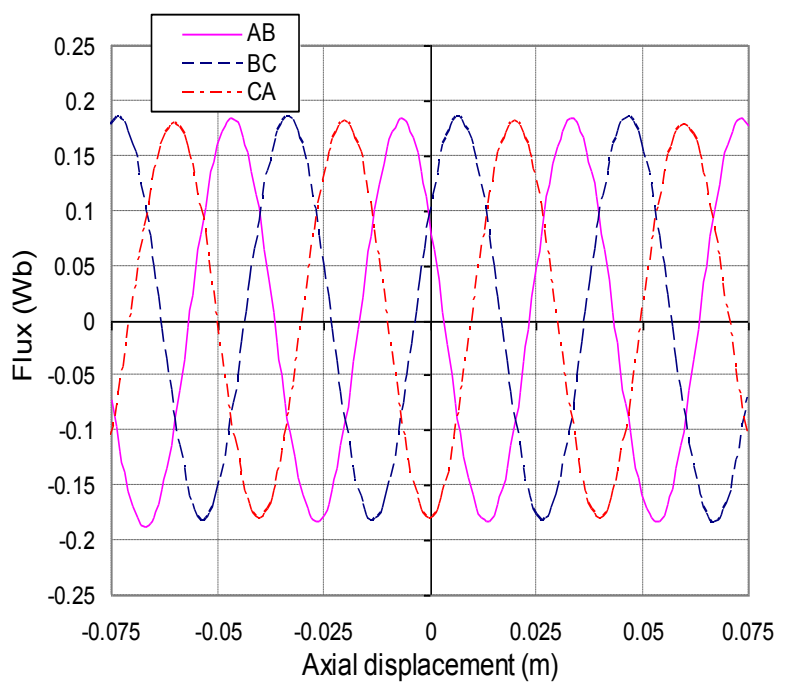

(b) Line-to-line flux linkages

Fig.4. Variation of measured phase and line-to-line flux linkage with axial displacement

The magnitude of flux-linkage in phase B is lower than that of the other two phases. This results from the fringing effect on phases $\mathrm{A}$ and $\mathrm{C}$ as they are located on the two sides while phase $\mathrm{B}$ is in the middle [12].

The measured and finite element (FE) predicted line-to- line flux-linkage of phases A and B are compared in Fig. 5. As can be seen, the magnitude of the measured flux linkage is $4 \%$ lower than the prediction. The causes of the reduction in the measurements will be discussed subsequently.

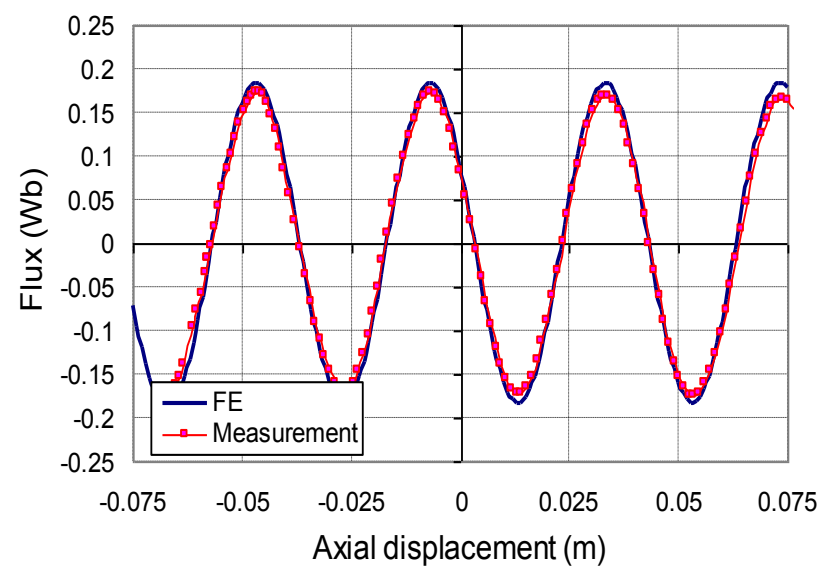

Fig. 5. Comparison of measured and predicated fluxlinkage in phase $\mathrm{AB}$

From the measured flux-linkage, the induced back-emfs per unit speed in each phase can be derived and they are shown in Fig. 6. It is evident that there is a small distortion in the back-emf waveforms of phases $\mathrm{A}$ and $\mathrm{C}$, which are caused by the end effect and fringing flux. This distortion is not visible in phase B back-emf waveform.

The variations of no-load cogging force and static frictional force with the mover axial displacement were measured using a load cell. The measurements were repeated by moving the magnet armature (mover) from the right to the left and vice versa.

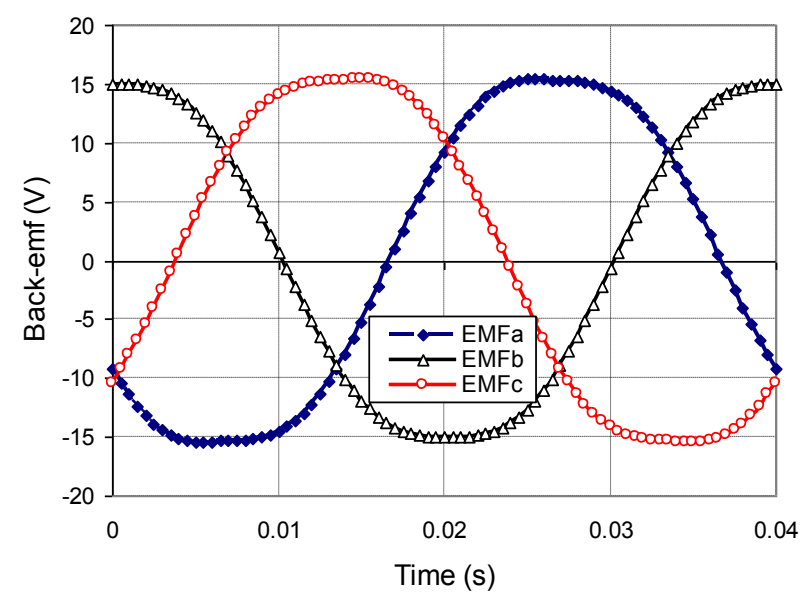

Fig. 6. Back-emf waveforms at $\mathrm{v}=1.0 \mathrm{~m} / \mathrm{s}$

The static frictional force can then be separated from the measured force-displacement characteristics. Next, the thrust force variations with the armature displacement when phase 
$\mathrm{AB}$ and phase $\mathrm{CA}$ were separately excited with 50A current were measured. Fig. 7 compares the measured and predicted thrust force variation with mover position.

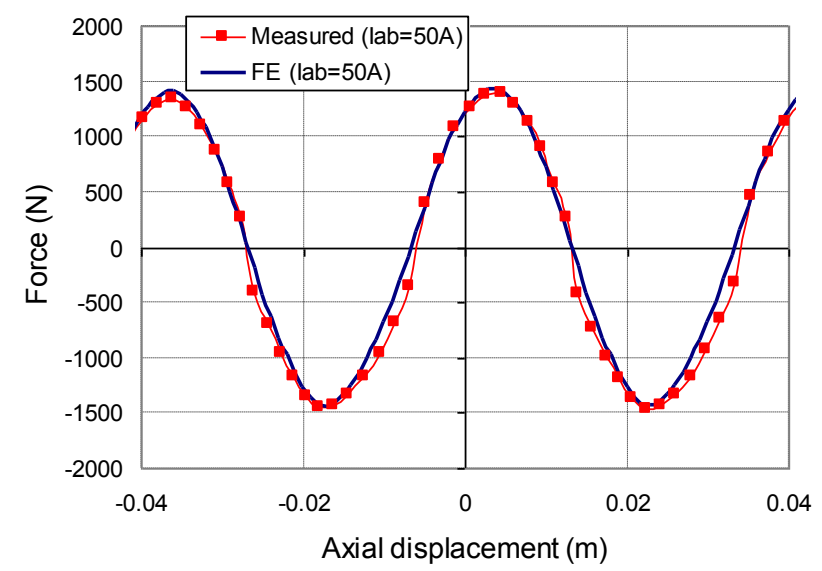

(a) phase $\mathrm{AB}$ excited with $50 \mathrm{~A}$ current

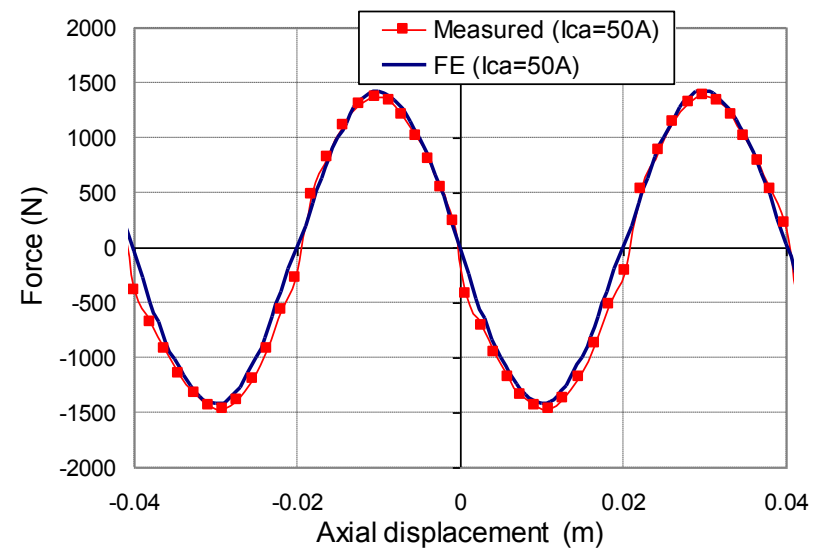

(b) phase CA excited with 50A current

Fig.7. Comparison of measured and predicated thrust force variation with mover displacement

The thrust force variation with current when all three phases are excited was also measured and the result is compared with the prediction in Fig. 8. The measurement was performed when the armature displacement was locked at zero while phase B was excited by current $I$ and phases A and $\mathrm{C}$ by $I / 2$. It should be noted that the measured thrust forces shown in Figs. 7 and 8 were obtained by subtracting the position dependent cogging and frictional force which was measured under open-circuit.

As will be seen from Figs. 4, 57 and 8, the measured peak flux linkage and thrust force are about $4 \%$ and $6 \%$ lower than that of prediction, respectively. This may be attributed to a number of factors. First, in the finite element (FE) prediction, the radially magnetized magnets are assumed to be ideal but in the prototype machine, they were replaced by 12 pieces of parallel magnetized magnets. This will result in leakage flux in circumferential direction and a small reduction in flux-linkage and thrust force which cannot be predicted using 2D axi-symmetrical model. Further, the tolerance on material properties, in particular the remanence of the magnets, may also lead to the discrepancy. In addition, the stress in the solid stator cores due to machining process may affect its $\mathrm{BH}$ curve, and hence contribute to the reduction in flux linkage and thrust force. Manufacturing tolerance and friction of the bearings will also contribute to the discrepancy between the prediction and measurement. Nevertheless, the thrust force varies almost linearly with current.

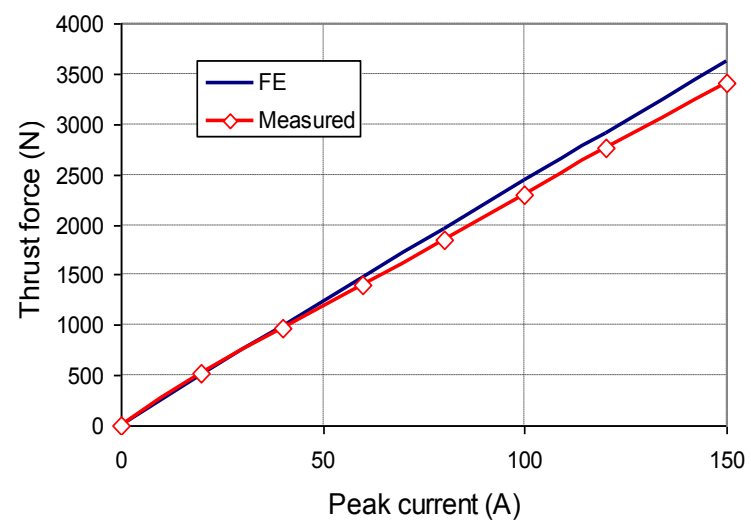

Fig. 8. Comparison of measured and predicated thrust force variation with current

\section{Thermal Modelling And Tests}

Assume that the thermal dissipation in the machine is predominant in the radial direction, the heat conduction in the machine may be represented by the transient thermal network shown in Fig. 9, where a half of the tooth pitch is modelled. $T_{s}, R_{i c}, R_{o c}, R_{h}$ and $R_{e}$ are the geometric parameters of the tooth, slot and back-iron. At the axial symmetrical surfaces of a tooth or a slot, the heat flows tangentially. The copper loss and iron loss can be dissipated both radially and axially via the representative thermal network. The thermal resistances, $R_{F 1}, R_{F 2}, R_{F 3}, R_{S 1}, R_{S 2}, R_{W 1}$ and $R_{W 2}$, of the model can be derived using the governing principle of the heat conduction, and are given in [10], where the heat sources, $p_{c u}$ and $p_{F e}$ are the copper loss and iron loss of the half slot-pitch stator. The iron loss $p_{F e}$ is relatively small compared with the copper loss and can be estimated analytically [13] or by finite element (FE) analysis [14] under no-load conditions. The thermal resistance which represents the heat convection at the stator surface is given by:

$$
R_{C}=1 /\left(\pi R_{e} T_{s} h_{c}\right)
$$

where $h_{c}$ is the thermal convection coefficient. $C_{w}$ and $C_{c}$ represent the thermal capacitance of the winding and stator core, respectively. The latter should also include the thermal mass of the stator case for simplicity. The thermal network in Fig. 9 can be further simplified to that shown in Fig. 10, where, 


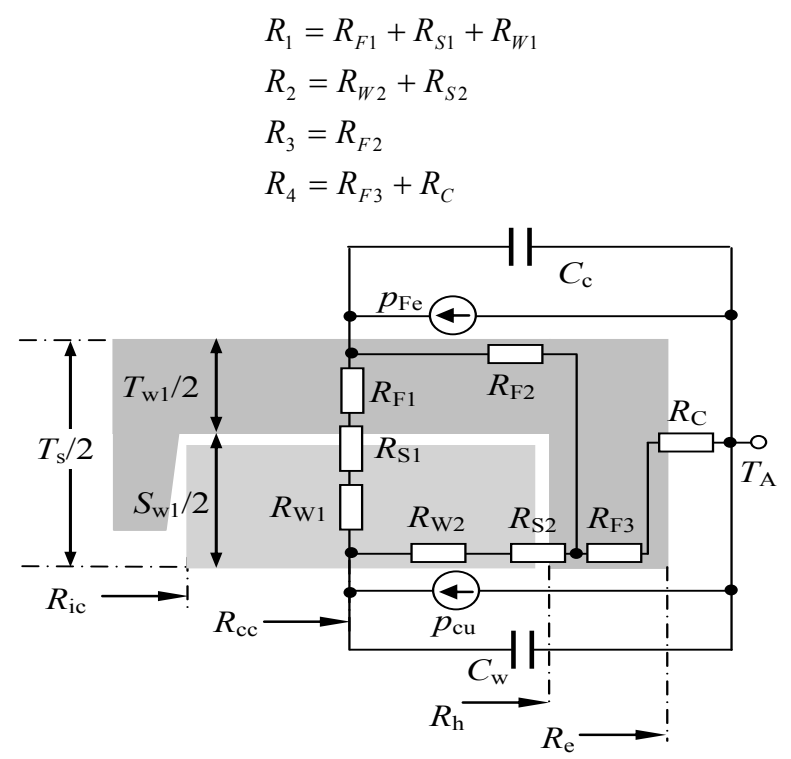

Fig. 9. Schematic of transient thermal model for tubular PM actuator

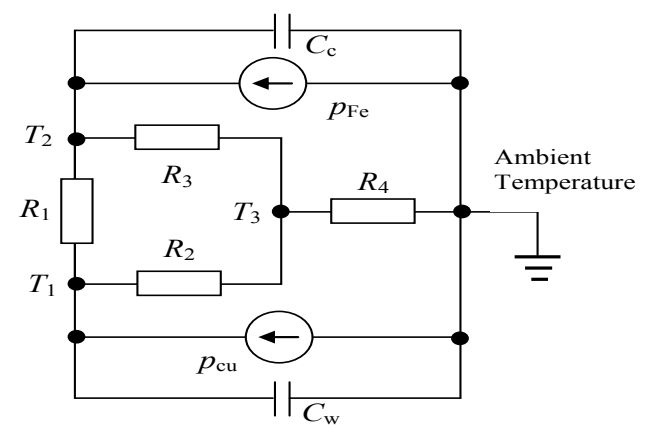

Fig. 10. Simplified transient thermal models

Thus the Laplace transformations of the temperature rises at the centres of the coil, the tooth and back-iron can be predicted by:

$$
\left[\begin{array}{c}
T_{1}(s) \\
T_{2}(s) \\
T_{3}(s)
\end{array}\right]=\left[\begin{array}{ccc}
G_{1}+G_{2}+s C_{w} & -G_{1} & -G_{2} \\
-G_{1} & G_{1}+G_{3}+s C_{c} & -G_{3} \\
-G_{2} & -G_{3} & G_{2}+G_{3}+G_{4}
\end{array}\right]^{-1}\left[\begin{array}{c}
p_{c u} \\
p_{F e} \\
0
\end{array}\right]
$$

where s denote the Laplace operator, and $G_{1} G_{2} G_{3}$ and $G_{4}$ are the inverses of $R_{1} R_{2} R_{3}$ and $R_{4}$, respectively. Assume that $I G A$ is the inverse of the complex thermal conductance matrix, the temperature rise, $T_{1}(\mathrm{t})$, at the centre of the coil can be determined by:
$T_{1}=L^{-1}\left\{\frac{1}{s}\left[I G A_{11}(s) p_{c u}+I G A_{12}(s) p_{F e}\right]\right\}$

where $L^{-1}$ denotes the inverse Laplace transformation. The copper loss in the half slot can be obtained by:

$$
p_{c u}=1.5 R I^{2} /\left(2 N_{s}\right)
$$

where $R$ is the winding resistance, $I$ the magnitude of phase current and $N_{s}$ is the number of slots.

Thermal tests under laboratory condition were also undertaken. The winding temperature was measured by thermal couplers inserted in the windings and the actuator was excited by a three phase current but the mover is lock at a fixed position. Figs. 11 (a) and (b) show the measured and predicted winding temperature variation with time when the three phase windings were excited with 60A current, without and with air-cooling, respectively. When the air-cooling was not present, the heat convection through the motor surface is much less effective, and for the same thermal mass, the thermal time constant is larger and the steady state temperature rise is higher. However, there will be some aircooling on the motor when the vehicle is travelling. The test with air cooling represents the measured air-flow under the worst condition when the vehicle is travelling at $20 \mathrm{mph}$. It is evident that the measured steady state temperature agrees very well with the predictions, which validates the thermal model.

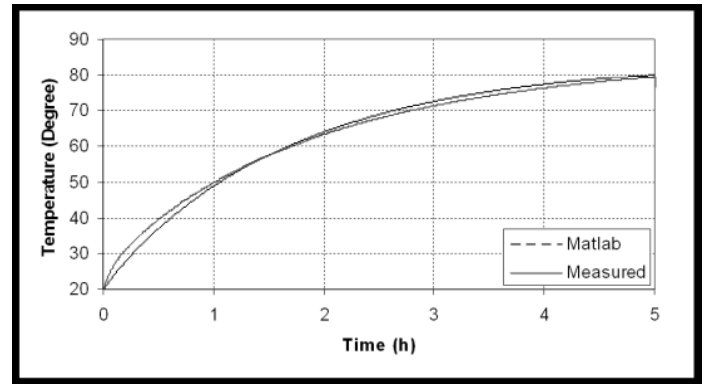

(a) without air cooling

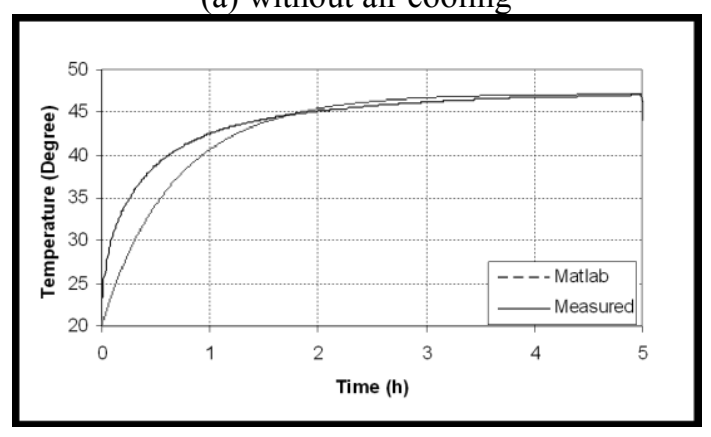

(b) with air-cooling

Fig. 11. Comparisons of measured and predicted temperature variation with time at $\mathrm{I}=60 \mathrm{~A}$. 


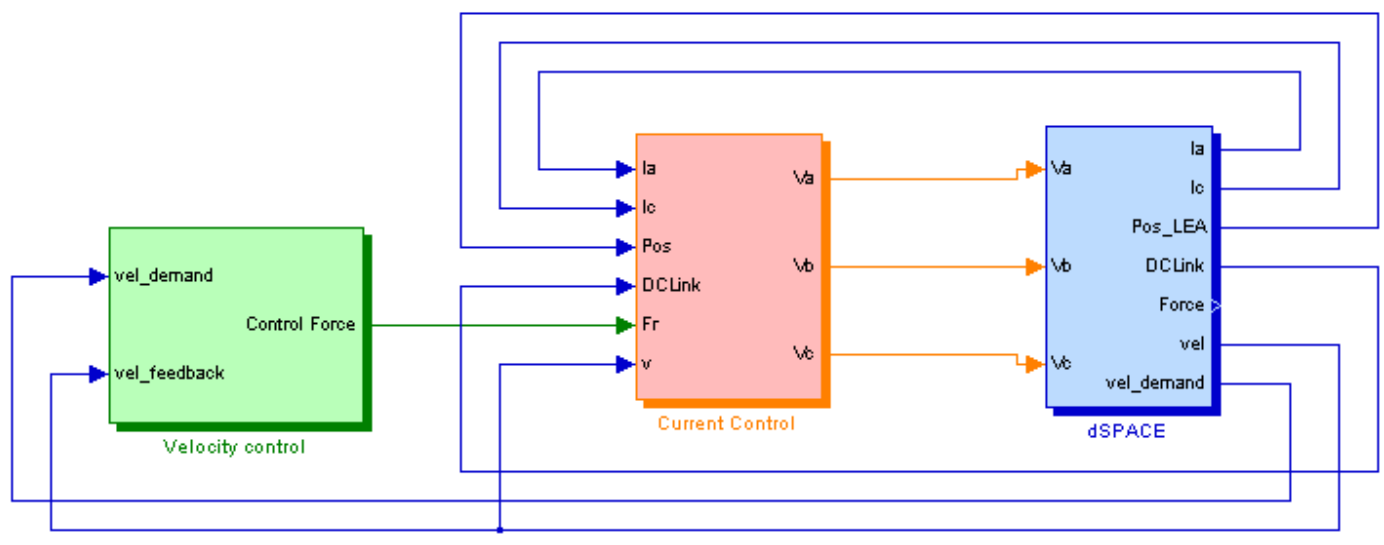

Fig. 12. Schematics of actuator control system

\section{Control of Actuator}

The actuator is controlled by a dSPACE system via a three phase inverter. Fig. 12 shows the schematic of the control system in which the functional blocks for active suspension control may also be included. The tests with a velocity controller which tracks a sinusoidal velocity demand will be reported in this paper. Under this condition, the actuator's load is effectively its own mass, damper (friction) and the integrated spring. The dSPACE is interfaced to the inverter via digital I/Os for PWM and condition monitoring, and analogue I/Os for current, position and DC link voltage feedbacks. The actuator position is measured by a LVDT and used for electronic commutation. The velocity feedback is obtained by filtering the position signal.

Both the velocity and current controllers are of PI type with the control bandwidths set to $10 \mathrm{~Hz}$ and $500 \mathrm{~Hz}$, respectively. Both the controllers implement anti-winding up protection. In the current control loop, a field weakening block, decoupling compensation and over-modulation are employed to improve dynamic performance and to extend the actuator operating range. The control system operates at a sampling rate of $10 \mathrm{kH}$ which equals to the switching frequency of the inverter.

Fig. 13 (a) shows the velocity tracking performance of the actuator control while the resultant displacement waveform is shown in Fig. 13 (b). As can be seen, the system can track the velocity demand reasonably well, albeit time varying steady state error exists. This is due to the fact that the PI controller is not capable of achieving zero tracking error for timevarying demand. In the active suspension control, the actuator control will mainly operate in force/current control mode.

Fig. 14 (a) shows the d-and q-axis currents as a result of the velocity tracking. For the mass-spring-damper system in which the actuator operates with a sinusoidal velocity, the required thrust force is sinusoidal. The q-axis current is effectively proportional to the thrust force, as is evident in Fig. 14 (a). Since the peak velocity is $\sim 1.0 \mathrm{~m} / \mathrm{s}$, no field weakening control is require and hence the d-axis current is virtually zero, apart from the current ripple due to PWM switching.

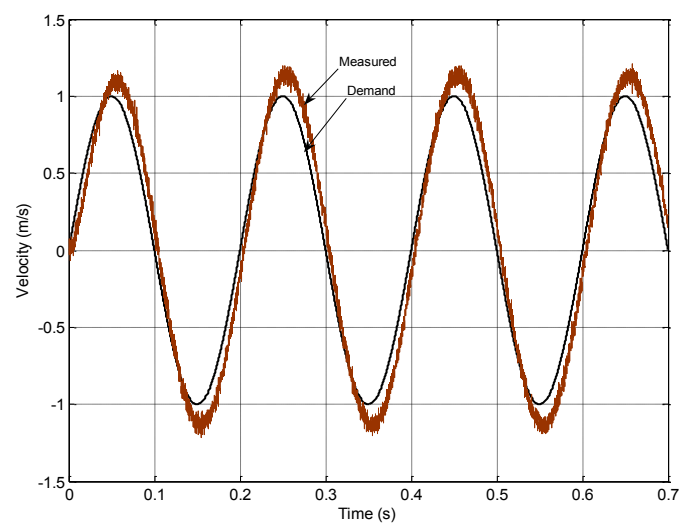

(a) Velocity demand and response

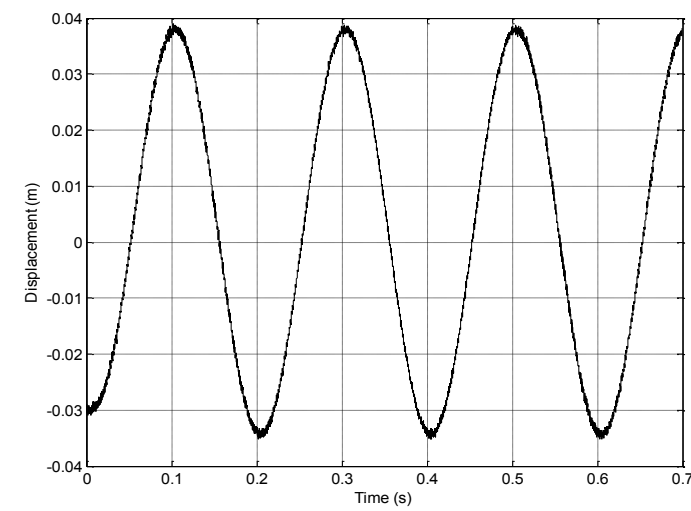

(b) displacement

Fig. 13. Velocity tracking performance

Fig. 14 (b) shows the phase current waveforms. It should be noted that the phase currents contain high order harmonics 
as a result of the reciprocating movement.
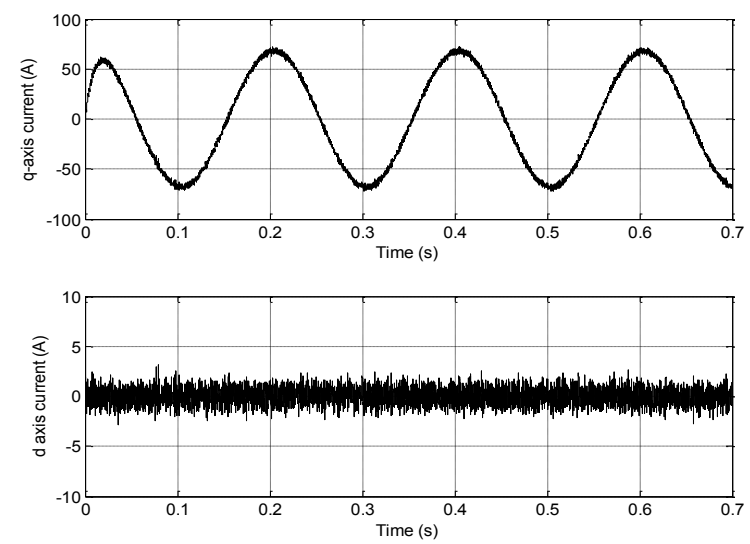

(a) d- and q-axis currents
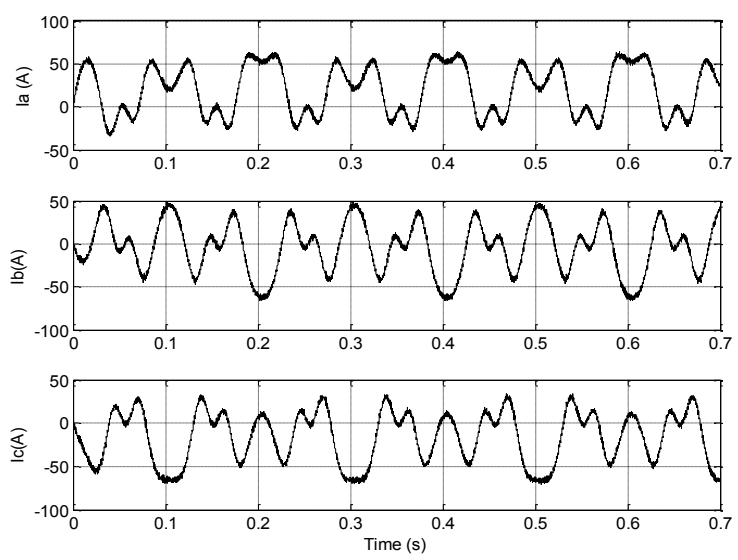

(b) phase currents

Fig. 14. Current waveforms

\section{Conclusions}

A linear permanent magnet actuator for active vehicle suspension has been described. The static, dynamic and thermal testing procedures have been presented and implemented for actuator characterization and for assessing its dynamic capability. It has been shown that the measured actuator parameters, such as back-emfs and thrust force are very close to their design predictions, although a small discrepancy exists which is attributable to a number of factors. The results from the temperature rise tests are consistent with the prediction by the derived transient thermal model. This provides a means of assessing the actuator thermal behavior under various operating conditions. The dynamic performance of the linear actuation system has been demonstrated by velocity tracking experiments.

\section{References}




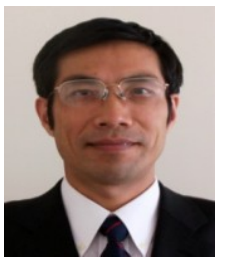

Jiabin Wang (SM'03) received the B.Eng. and M.Eng. degrees from Jiangsu University of Science and Technology, Zhengjiang, China, in 1982 and 1986, respectively, and the Ph.D. degree from the University of East London, London, U.K., in 1996, all in electrical and electronic engineering. Currently, he is a Professor in Electrical Engineering at the University of Sheffield, Sheffield, U.K. From 1986 to 1991, he was with the Department of Electrical Engineering at Jiangsu University of Science and Technology, where he was appointed a Lecturer in 1987 and an Associated Professor in 1990. He was a Postdoctoral Research Associate at the University of Sheffield, Sheffield, U.K., from 1996 to 1997, and a Senior Lecturer at the University of East London from 1998 to 2001. His research interests range from motion control to electromagnetic devices and their associated drives for applications in automotive, household appliances and aerospace sectors.

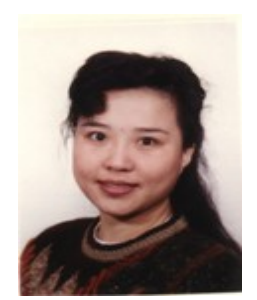

Weiya Wang received the B.Eng. degree from Jiangsu University of Science and Technology, China, in 1982 and the M.Sc. degree from the University of East London in 1996, and the $\mathrm{PhD}$ degree from the University of Sheffield in 2006, all in electrical and electronic engineering.

Currently, she is a Senior Research Fellow at the University of Sheffield. From 1982 to 1993 she worked in the Department of Electrical Engineering, Jiangsu University of Science and Technology, being appointed a lecturer in 1988. From 1997 to 2008, she was a Research Associate at the University of Sheffield and a Senior Research Fellow at Anglia Polytechnic University. She was Design Engineer at Magnomatics Ltd, U.K., from 2009 to 2010. Her research interests include rotary, linear and multiple degree-of-freedom motors and actuators, and their control. 